\title{
High-luminosity LHC prospects with the upgraded ATLAS detector
}

\author{
Magdalena Slawinska*† \\ Nikhef National institute for subatomic physics \\ E-mail: Magdalena.Slawinska@nikhef.nl
}

Run 1 at the LHC was very successful with the discovery of a new boson. The boson's properties are found to be compatible with those of the Standard Model Higgs boson. It is now revealing the mechanism of electroweak symmetry breaking and (possibly) the discovery of physics beyond the Standard Model that are the primary goals of the just restarted LHC. The ultimate precision will be reached at the High-Luminosity LHC phase with a proton-proton centre-of-mass energy of 14 $\mathrm{TeV}$. In this contribution physics prospects are presented for ATLAS for the integrated luminosities 300 and $3000 \mathrm{fb}^{-1}$ : the ultimate precision attainable on measurements of the Higgs boson couplings to elementary fermions and bosons, its trilinear self-coupling, as well as perspectives on the searches for supersymmetric partners associated with the Higgs boson. Benchmark studies are presented to show how the sensitivity improves at the future LHC runs. For all these studies, a parameterised simulation of the upgraded ATLAS detector is used and expected pileup conditions are accounted for.

XXIV International Workshop on Deep-Inelastic Scattering and Related Subjects 11-15 April, 2016

DESY Hamburg, Germany

\footnotetext{
* Speaker.

${ }^{\dagger}$ On behalf of the ATLAS Collaboration.
} 


\section{Introduction}

During Run 1 of the LHC a new boson was discovered by the ATLAS and CMS collaborations [1,2]. Its measured properties are largely compatible with those of the SM Higgs boson [3] and precise measurements are instrumental in determining if it is indeed "the" Higgs predicted by [4] and responsible for electroweak symmetry breaking.

After a very successful Run 1 of the LHC, Run 2 data taking is currently taking place with proton-proton collisions at a centre-of-mass energy $\sqrt{s}=13 \mathrm{TeV}$. ATLAS is expected to collect an integrated luminosity of about $100 \mathrm{fb}^{-1}$ by the end of 2018. Starting in 2021, proton-proton collisions at $\sqrt{s}=14 \mathrm{TeV}$ are expected during the Run 3 of the LHC amounting to about $300 \mathrm{fb}^{-1}$. In 2026 the High Luminosity (HL-LHC) phase will start. With an instantenuous luminosity of $7.5 \times 10^{34} \mathrm{~cm}^{-2} \mathrm{~s}^{-1}$ about $3000 \mathrm{fb}^{-1}$ are expected to be collected over a period of about ten years. Collisions at $14 \mathrm{TeV}$ will be recorded with up to $\mu=200$ proton-proton interactions per bunch crossing.

In order to cope with a threefold increase of $\mu$ with respect to Run 2 and to enable efficient pileup mitigation, all detector subsystems must be upgraded or replaced. The planned upgrade is described in the Letter of Intent [6], where various layout options are considered, and summarised in the Scoping Document [7] (2015), where the final layout was chosen based on desirable physics capabilities. In the trigger and data acquisition system a two-level hardware trigger with L0 output rate up to $1 \mathrm{MHz}$ and L1 up to $400 \mathrm{kHz}$ is foreseen together with a High-Level Trigger with 10 $\mathrm{kHz}$ output (permanently recorded data). "Custom hardware" triggers will enable data streaming at rates 1-40 MHz. The inner tracker itself will be replaced with a new, all-silicon detector. Its pseudorapidity coverage will be increased from the current $|\eta| \leq 2.4$ to $|\eta| \leq 4$ by the addition of four pixel discs in the forward region. Since calorimeter cell noise (electronic and pileup) increases with pseudorapidity $|\eta|$, detector improvements in the forward regions are necessary. The Liquid Argon (LAr) forward electromagnetic calorimeter will be replaced by a higher granularity detector (sFcal). Additionally, a High Granularity Timing Detector will be installed in front of LAr end-caps, in the pseudorapidity region $2.4 \leq|\eta| \leq 4.3$. Readout electronics of LAr and Tile Calorimeters will be replaced due to forseen radiation damage in Run 3. For the muon system addition of new Resistive Plate Chambers (RPCs) in the barrel is expected.

In these proceedings I will compare physics prospects for the HL-LHC with prospects for 300 $\mathrm{fb}^{-1}$ integrated luminosity expected at Run 3 . Section 2 will decribe the procedure of extracting the SM Higgs coupling strengths mentioning assumptions behind different fits. I will point out the role of theoretical uncertainties in improving the precision on coupling extraction. In section 3 searches for signs of BSM physics in Higgs couplings will be outlined. In section 4 I will discuss prospects for observing Higgs pair production and measuring Higgs trilinear self-coupling. An example of a search for supersymmetric particles will be discussed in section 5 .

The physics studies described in these proceedings have been performed in the period 20142015 , hence not all improvements of the upgraded detector were taken into account in every analysis. Furthermore, some analyses assume earlier estimates on instantenuous luminosity and $\mu=140$. 


\section{Measurement of SM Higgs couplings}

The HL-LHC provides an opportunity to test the Standard Model with precise measurements of the Higgs boson properties. ATLAS analysed the following channels: $h \rightarrow \mu \mu, h \rightarrow \tau \tau, h \rightarrow Z Z$, $h \rightarrow W W, h \rightarrow \gamma \gamma(0 / 1 / 2$ jet categories, inclusive), $h \rightarrow Z \gamma, V h, t \bar{t} h \rightarrow \gamma \gamma, V h \rightarrow b \bar{b}$ [8]. The precision for each of the Higgs boson production and decay categories $(\Delta \mu / \mu)$ is presented in the left panel in Fig. 1. Green bars correspond to $300 \mathrm{fb}^{-1}$ and dark blue bars correspond to $3000 \mathrm{fb}^{-1}$. The highest precision of $\sim 5 \%$ can be reached with $3000 \mathrm{fb}^{-1}$ in di-boson final states. An experimental precision of $\sim 4 \%$ is reachable for the dominanant producion mode, the gluon fusion. The largest gains in precision between 300 and $3000 \mathrm{fb}^{-1}$ is observed in rare channels: production associated with vector bosons and associated with $t \bar{t}$ because these channels are statistically dominated.

The measurements of signal strengths are interpreted as the SM LO Higgs couplings to bosons and fermions. Assuming the decay width of the Higgs boson to be zero, the coupling strengths in its production and decay can be factorised. Deviations from the SM Higgs production cross-sections $\sigma$ and its branching ratios $B$ are denoted as $\kappa_{i}$ and $\kappa_{j}$ in the following way:

$$
\frac{\sigma(i i \rightarrow h) B(h \rightarrow j j)}{\sigma_{S M}(i i \rightarrow h) B(h \rightarrow j j)_{S M}}=\frac{\kappa_{i}^{2} \kappa_{j}^{2}}{\kappa_{h}^{2}},
$$

where $\kappa_{h}$ denotes the scaling of the SM Higgs width $\Gamma_{h}$. All $\kappa_{i}$ parameters correspond to LO degrees of freedom.

There are large uncertainties related to: $\Gamma_{h}$, assumptions on Higgs branching ratios to invisible particles and other model-dependent theoretical and experimental assumptions that enter in the coupling fit. To avoid them some parametrisations use ratios $\lambda_{i j}=\frac{\kappa_{i}}{\kappa_{j}}$. The expected precision on these ratios are shown in the right panel in Fig. 1.

In the minimal coupling fit it is assumed that all Higgs couplings to bosons, $\kappa_{V}$, and fermions, $\kappa_{f}$, are identical. In this model the expected experimental precision with $300 \mathrm{fb}^{-1}$ can reach $\sim 2.5 \%$ on $\kappa_{V}$ and $\sim 7.1 \%$ on $\kappa_{f}$. Including current theory uncertainties the total uncertainties of $\sim 4.3 \%$ and $\sim 8.8 \%$ are expected. The largest reduction of uncertainties with the increased luminosity is expected for $\kappa_{f}$, where an experimental precision of $\sim 3.2 \%$ can be reached $(\sim 5.1 \%$ including current theory uncertainties) compared to $\kappa_{V} \sim 1.7 \%$ ( $\sim 3.3 \%$ including theory uncertainties).

\section{BSM Physics in Higgs couplings}

With the estimated precision of measuring Higgs couplings at 300 and $3000 \mathrm{fb}^{-1}$ constraints on theories that extend the Standard Model are investigated [9]: an additional electroweak singlet, an additional electroweak doublet (i.e. the two-Higgs-doublet model), a composite Higgs boson, a simplified Supersymmetric Standard Model (MSSM), and a Higgs portal to dark matter. I will highlight one of these results here: tests of models predicting Higgs compositeness.

If the Higgs is not an elementary particle but a composite pseudo-Goldstone boson its couplings to bosons and fermions will deviate from the SM predictions. These deviations can be parametrised in terms of a Higgs compositeness scale $F$ and Higgs vacuum expectation value $v$ : $\xi=v^{2} / F^{2}$. In the $\mathrm{SM} \xi$ is zero as $F \rightarrow \infty$. Simple models predicting a composite Higgs boson 
ATLAS Simulation Preliminary

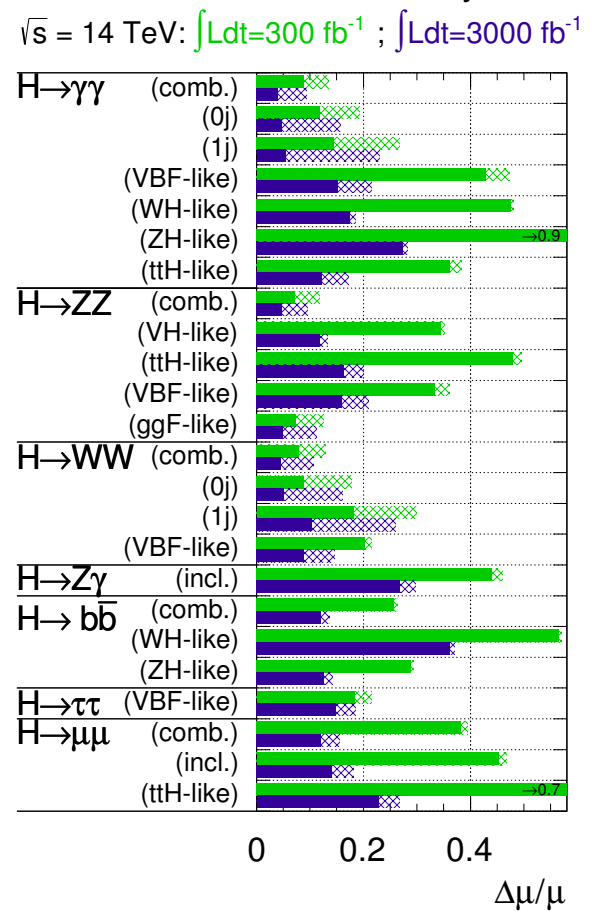

ATLAS Simulation Preliminary $\sqrt{\mathrm{S}}=14 \mathrm{TeV}: \int \mathrm{Ldt}=300 \mathrm{fb}^{-1} ; \int \mathrm{Ldt}=3000 \mathrm{fb}^{-1}$

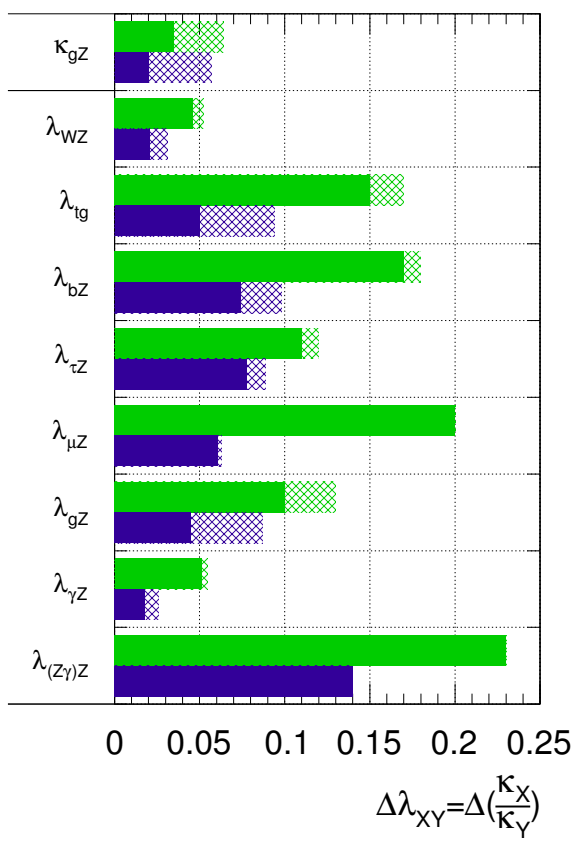

Figure 1: The uncertainties of the Higgs signal strengths in different categories (left) and couplings ratios $\lambda_{i j}$ (right). Figures taken from [8].

\begin{tabular}{|crr|rr|}
\hline Model & & $\begin{array}{r}300 \mathrm{fb}^{-1} \\
\text { All unc. }\end{array}$ & $\begin{array}{r}3000 \mathrm{fb}^{-1} \\
\text { No theor. unc. }\end{array}$ & $\begin{array}{r}\text { All unc. } \\
\text { No theor. unc. }\end{array}$ \\
\hline MCHM4 & 620 & 810 & 710 & 980 \\
MCHM5 & 780 & 950 & 1000 & 1200 \\
\hline
\end{tabular}

Table 1: Expected 95\% CL lower limits on Higgs compositeness scale $F$ in GeV. Table taken from [9].

include MCHM4 and MCHM5 [10]. In MCHM4 the ratios of the Higgs boson couplings to bosons and fermions to their SM expectations are equal and can be parametrised by

$$
\kappa_{V}=\kappa_{f}=\sqrt{1-\xi}
$$

MCHM5 predicts

$$
\kappa_{V}=\sqrt{1-\xi}, \quad \kappa_{f}=\frac{1-2 \xi}{\sqrt{1-\xi}}
$$

A comparison of exclusion powers for these models, assuming the SM Higgs boson, for the Run 3 and HL-LHC data is presented in Table 1.

\section{Higgs Pair Production}

A direct insight into the mechanism of electroweak symmetry breaking could be gained by the 
measurement of Higgs potential $V$. In the Standard Model after electroweak symmetry breaking

$$
V=\frac{m_{h}^{2}}{2} h^{2}+\lambda_{h h h} h^{3}+\frac{\lambda_{h h h h}^{4}}{4} h^{4}
$$

The Higgs boson acquires triple and quartic self-couplings, fully determined by the Higgs mass $m_{h}$ and vacuum expectation value $v: \lambda_{h h h}=m_{h}^{2} /(2 v)$ and $\lambda_{h h h h}=m_{h}^{2} /\left(2 v^{2}\right)$. Any deviations from these values will indicate that electroweak symmetry breaking is triggered by a more complex physical mechanism than that of Ref. [4].

The direct measurement of Higgs trilinear (quartic) coupling involves the observation of two (three) Higgs bosons in the final state. The cross-section of di-Higgs production in the dominant gluon-gluon fusion production mode is small $\sigma^{N N L O+N N L L}=39.56 \mathrm{fb}$ [11] The tri-Higgs production cross-section yields only $\sigma^{\text {NLOFTapprox }}=0.09 \mathrm{fb}$ [12]. Therefore di-Higgs production will probably be observed for the first time at the HL-LHC, while tri-Higgs production is beyond its reach. ATLAS has performed two searches for the SM Higgs pair production: in the $b \bar{b} \gamma \gamma$ [13], $b \bar{b} \tau \tau[14]$ channels.

The $b \bar{b} \gamma \gamma$ channel benefits from good mass resolution of the Higgs decaying to two photons and from relatively small backgrounds. The challenge of this analysis lies in small signal yields: at the HL-LHC about 300 events are expected. In addition to irreducible backgrounds - continuum $b \bar{b} \gamma \gamma$, single Higgs: $t \bar{t} H(\rightarrow \gamma \gamma), Z(\rightarrow b \bar{b}) H(\rightarrow \gamma \gamma)$ and $b \bar{b} H(\rightarrow \gamma \gamma)$ - the signal is accompanied by reducible backgrounds from light-flavour jets misidentified as $b$-jets and electrons misidentified as photons. This analysis assumes $\mu=140$ and estimates the upgraded ATLAS performance for measuring the $\eta$ and $p_{T}$ of jets and photons with the use of the $\eta / p_{T}$-dependent efficiency and resolution functions. The transverse momenta of the jets are smeared by $10-25 \%$. The performance of b-tagging for these jets is simulated by applying an efficiency function, corresponding to a mean efficiency of $70 \%$. To account for radiation of partons outside the jet cone and semi-leptonic $b$ hadron decays a correction of jet energy is applied. The identification of photons is modelled with a plateau efficiency equal to $76 \%$ for photons with transverse momenta larger than $80 \mathrm{GeV}$. The rates of jets being misreconstructed as photons, light-flavour jets as $b$-jets and $c$-jets as $b$-jets are assumed to be equal to $0.25 \%, 1 \%$, and $30 \%$, respectively. In the event selection two energetic, isolated photons and two b-jets are required. The invariant mass of two photons (two b-jets) must fulfill the requirement $123 \mathrm{GeV}<m_{\gamma \gamma}<128 \mathrm{GeV}\left(100 \mathrm{GeV}<m_{b \bar{b}}<150 \mathrm{GeV}\right)$ and transverse momenta $p_{T}^{\gamma \gamma}, p_{T}^{b \bar{b}}>110 \mathrm{GeV}$. The effect of systematic uncertainties is neglected in this analysis. The expected limits set on the value of the Higgs trilinear coupling $\lambda$ (assuming no other sources of new physics) are $\lambda / \lambda_{S M} \in[-1.3,8,7]$ at $95 \%$ C.L..

The $b \bar{b} \tau \tau$ channel has the third largest di-Higgs branching ratio of $\sim 7.3 \%$. The search in this channel is performed in three subchannels defined by decay modes of the $\tau$ leptons: di-leptonic, semi-leptonic and hadronic. This analysis assumes $\mu=200$ and uses a track a confirmation algorithm to suppress pile-up. The dominant backgrounds are the production of $t \bar{t}, W+$ jet, $Z+$ jet, di-bosons and multijet events. The largest sensitivity is obtained in the hadronic subchannel, resulting in an upper limit on the Higgs pair production cross-section equal to 4.3 times its SM value at $95 \%$ C.L. The corresponding expected exclusion limits on $\lambda_{\text {hhh }}$ in all three subchannels are $\lambda / \lambda_{S M} \in[-4,12]$. 
Higgs pair production can also probe the presence of $\mathrm{TeV}$ scale resonances interacting with the Higgs, such as Kaluza-Klein gravitons. Such a resonant production of a pair of Higgs bosons decaying to $b \bar{b} b \bar{b}$ was studied in [7]. The HL-LHC study found a $4.4 \sigma$ expected significance for the graviton with a mass of $2 \mathrm{TeV}$. The largest gain in sensitivity between different inner tracker layouts considered in the Scoping Document stems from an inproved $b$-tagging performance.

\section{Prospects for discovering supersymmetric electro-weakinos}

A possible explanation of the electroweak symmetry breaking explored by ATLAS is through a supersymmetric extension of the Standard Model. This theory predicts the existence of many new particles, among them charginos $\tilde{\chi}_{1}^{ \pm}$and neutralinos $\tilde{\chi}_{2}^{0}$, the mass eigenstates from superposition of the supersymmetric Higgs partners and electroweak gauge bosons. The ATLAS sensitivity to detecting electro-weakinos in the mass range of several hundreds $\mathrm{GeV}$ was studied in a simplified Wh-mediated model $[15,16]$, in which $\tilde{\chi}_{1}^{ \pm}$and $\tilde{\chi}_{2}^{0}$ are pair-produced and decay to the $\mathrm{W}$ boson and the lightest neutralino $\tilde{\chi}_{1}^{0}$, Higgs boson and $\tilde{\chi}_{1}^{0}$, respectively. The studied experimental signature consists of a lepton, a pair of b-quarks and missing energy due to undetected neutrino and neutralinos.

In this analysis $\mu=60(\mu=140)$ was assumed for Run 3 (HL-LHC). With the $300 \mathrm{fb}^{-1}$ (3000 $\left.\mathrm{fb}^{-1}\right)$ ATLAS is sensitive to electro-weakinos down to $1.1 \mathrm{TeV}(800 \mathrm{GeV})$ mass range. The improvement in the HL-LHC phase is driven by increase in b-tagging efficiency due to upgrades in the inner tracker and calorimeter.

\section{Conclusions}

Understanding electroweak symmetry breaking requires precise measurements in the Higgs sector. In order to accomplish this task in the challenging environment of the HL-LHC extensive studies of physics prospects are carried out to optimise the planned detector upgrades.

Higgs couplings to bosons and fermions can be measured at HL-LHC with uncertainties up to two times smaller than with $300 \mathrm{fb}^{-1}$. The largest improvements are expected in rare $\mathrm{VH}$ and $\mathrm{ttH}$ production channels. The precision of measurements is limited by theoretical uncertainties of the gluon fusion production cross-section. These measurements are also interpreted in the context of BSM models. In the MCHM5 discussed in these proceedings the lower limit on the Higgs compositeness scale can be set at $1 \mathrm{TeV}$ with $3000 \mathrm{fb}^{-1}$, compared to $780 \mathrm{GeV}$ with $300 \mathrm{fb}^{-1}$.

The direct probe of the Higgs potential - di-Higgs production - could be observed for the first time at the HL-LHC even in the rare $b \bar{b} \gamma \gamma$ channel. In the final states with $b \bar{b} \gamma \gamma$ and $b \bar{b} \tau \tau$ the $\mathscr{O}(1)$ limits can be set on Higgs trilinear coupling $\lambda_{\text {hhh }}$.

The discovery potential of direct searches for New Physics interacting with the Higgs will be extended. Sensitivity to Kaluza-Klein gravitons reaches up to $2 \mathrm{TeV}$. ATLAS is sensitivie to electro-weakinos in the $800 \mathrm{GeV}$ mass range. For all analyses theory improvements are crucial in obtaining higher precision. 


\section{References}

[1] ATLAS Collaboration, Observation of a new particle in the search for the Standard Model Higgs boson with the ATLAS detector at the LHC, Phys. Lett. B716 (2012) 1-29, [arXiv:1207.7214].

[2] CMS Collaboration, Observation of a new boson at a mass of $125 \mathrm{GeV}$ with the CMS experiment at the LHC, Phys. Lett. B716 (2012) 30-61, [arXiv:1207.7235].

[3] ATLAS and CMS Collaborations, Measurements of the Higgs boson production and decay rates and constraints on its couplings from a combined ATLAS and CMS analysis of the LHC pp collision data at $\sqrt{s}=7$ and $8 \mathrm{TeV}$, Tech. Rep. arXiv:1606.02266. CERN-EP-2016-100, ATLAS-HIGG-2015-07, CMS-HIG-15-002, CERN, Geneva, Jun, 2016.

[4] P. W. Higgs, Broken symmetries, massless particles and gauge fields, Phys.Lett. 12 (1964) 132-133, P. W. Higgs, Broken Symmetries and the Masses of Gauge Bosons, Phys.Rev.Lett. 13 (1964) 508Đ509. F. Englert and R. Brout, Broken Symmetry and the Mass of Gauge Vector Mesons, Phys.Rev.Lett. 13 (1964) $321 Đ 323$.

[5] ATLAS Collaboration, The ATLAS Experiment at the CERN Large Hadron Collider, 2008 JINST 3 S08003.

[6] ATLAS Collaboration,Letter of Intent for the Phase-II Upgrade of the ATLAS Experiment, Tech. Rep. CERN-LHCC-2012-022. LHCC-I-023, CERN, Geneva, Dec 2012, https://cds.cern.ch/record/1502664.

[7] ATLAS Collaboration, ATLAS Phase-II Upgrade Scoping Document, Tech. Rep. CERN-LHCC-2015-020. LHCC-G-166, CERN, Geneva, Sep, 2015, https://cds.cern.ch/record/2055248.

[8] ATLAS Collaboration, Projections for measurements of Higgs boson signal strengths and coupling parameters with the ATLAS detector at a HL-LHCTech. Rep. ATL-PHYS-PUB-2014-016, CERN, Geneva, Oct. 2014, https://cds.cern.ch/record/1956710.

[9] ATLAS Collaboration, Prospects for New Physics in Higgs Couplings Studies with the ATLAS Detector at the HL-LHC Tech. Rep. ATL-PHYS-PUB-2014-017, CERN, Geneva, Oct. 2014, https://cds.cern.ch/record/1956711.

[10] K. Agashe, R. Contino, and A. Pomarol, The minimal composite higgs model, Nuclear Physics B 719 (2005), no. 12165 Đ 187.

[11] D. de Florian and J. Mazzitelli, Higgs pair production at next-to-next-to-leading logarithmic accuracy at the LHC, JHEP 09 (2015) 053, arXiv:1505.07122 [hep-ph]. D. de Florian and J. Mazzitelli, Higgs Boson Pair Production at Next-to-Next-to-Leading Order in QCD, Phys. Rev. Lett. 111 (2013) 201801, arXiv:1309.6594 [hep-ph]. S. Borowka, N. Greiner, G. Heinrich, S. P. Jones, M. Kerner, J. Schlenk, U. Schubert, and T. Zirke, Higgs boson pair production in gluon fusion at NLO with full top-quark mass dependence, arXiv:1604.06447 [hep-ph].

[12] F. Maltoni, E. Vryonidou, and M. Zaro, Top-quark mass effects in double and triple Higgs production in gluon-gluon fusion at NLO, JHEP 11 (2014) 079, arXiv:1408.6542 [hep-ph].

[13] ATLAS Collaboration, Prospects for measuring Higgs pair production in the channel $H(\rightarrow \gamma \gamma) H(\rightarrow b \bar{b})$ using the ATLAS detector at the HL-LHC, Tech. Rep. ATL-PHYS-PUB-2015-046, CERN, Geneva, Nov. 2015, https://cds.cern.ch/record/1956733.

[14] ATLAS Collaboration, Higgs Pair Production in the $H(\rightarrow \tau \tau) H(\rightarrow b \bar{b})$ channel at the High-Luminosity LHC, Tech. Rep. ATL-PHYS-PUB-2015-046, CERN, Geneva, Oct. 2014, https://cds.cern.ch/record/2065974. 
[15] ATLAS Collaboration, Search for Supersymmetry at the high luminosity LHC with the ATLAS experiment, Tech. Rep. ATL-PHYS-PUB-2014-010, CERN, Geneva, Jul. 2014, https://cds.cern.ch/record/1735031.

[16] ATLAS Collaboration, Prospect for a search for direct pair production of a chargino and a neutralino decaying via a $W$ boson and the lightest Higgs boson in final states with one lepton, two $b$-jets and missing transverse momentum at the high luminosity LHC with the ATLAS Detector., Tech. Rep. ATL-PHYS-PUB-2015-032, CERN, Geneva, Jul. 2015, https://cds.cern.ch/record/2038565 